\title{
Évolution saisonnière des peuplements phytoplanctoniques dans le lac-réservoir El Kansera (Maroc), en relation avec certains paramètres abiotiques et biotiques
}

\section{Seasonal evolution of phytoplanktonic communities in relation to abiotic and biotic parameters in El Kansera lake-reservoir (Morocco)}

\author{
Fqih Berrada D. ${ }^{*(1)}$; Berrada R. ${ }^{(2)}$; Benzekri A. ${ }^{(3)}$; Jabry E..$^{(3)}$
}

(1)* Etudiante chercheur à la Faculté des Sciences Aï Chock de Casablanca (Département Biologie, B.P : 5366), et au Laboratoire Public d'Essais et d'Etudes (Centre d'Eludes et de Recherche sur l'Environnement et la Pollution):

(2) Faculté des Sciences Ain Chock de Casablanca (UFR: Biologie et Santé);

(3) Laboratoire Public d'Essais et d'Etudes (Centre d'Etudes et de Recherche sur l'Environnement et la Pollution).

Résumé. - L'étude de l'évolution saisonnière des peuplements phytoplanctoniques dans le lac-réservoir El Kansera vise à comprendre le phénomène d'eutrophisation de cet écosystème lacustre marocain. La biomasse (poids frais en $\mathrm{mg} / \mathrm{l}$ et chlorophylle a en $\mu \mathrm{g} / \mathrm{l}$ ) et la densité algale ont été suivies durant deux années consécutives (1995 et 1996), marquées par de fortes variations climatiques. Durant la première année d'étude (année de sécheresse), le pic algal se situe au printemps-été (73 mg/l en juin); il est dominé en biomasse par la Cyanobactérie Microcystis aeruginosa. La chlorophylle a atteint un maximum de $40 \mu \mathrm{g} / \mathrm{l}$. L'année suivante (année pluvieuse), le peuplement algal est plus diversifié, suite à l'enrichissement du milieu en sels nutritifs : Diatomées (Cyclotella ocellata) et Chlorococcales du genre Crucigenia et Scenedesmus se succèdent au printemps. Mais en été, la Cyanobactérie Microcystis aeruginosa recolonise le milieu avec une biomasse de $72,7 \mathrm{mg} / \mathrm{l}$. Les variables environnementales (climat, physicochimie de l'eau et apports exogènes de l'oued Beht notamment) semblent régir de près la succession phytoplanctonique dans la retenue EI Kansera. Le zooplancton régule peu la production algale (de Microcystis notamment), en raison de la grande taille de cette espèce et de son caractère toxique. Selon le modèle de l'O.C.D.E (1982), la retenue passe d'un stade hypereutrophe en 1995 à un stade méso-eutrophe en 1996, avec un retour à l'eutrophie en saison d'été.

Mots-clés. - phytoplancton, Cyanobactéries, Diatomées, biomasse, chlorophylle, zooplancton, lac-réservoir, eutrophisation, Maroc. 
Abstract. - A study of the seasonal evolution of phytoplankton in El Kansera lake-reservoir was performed in order to understand the eutrophication of this Moroccan lacustrian ecosystem. The biomass (wet weight in $\mathrm{mg} / \mathrm{l}$ and chlorophyll a in $\mu \mathrm{g} / \mathrm{l}$ ) and algal density were monitored for two successive years, characterized by significant climatic fluctuations. During the first study year (dry), the algal peak occurred in spring-summer $(73 \mathrm{mg} / \mathrm{l}$ in June); it was dominated by the Cyanobacteria Microcystis aeruginosa. Chlorophyll a reached a maximum of $40 \mu \mathrm{g} / \mathrm{l}$. The following year (rainy), phytoplankton was more diversified due to the water's enrichment by nutrients: Diatoms (Cyclotella ocellata) and Chlorococcals (Crucigenia and Scenedesmus) appeared in spring. In summer, Microcystis aeruginosa recolonized the reservoir with a biomass about of $72.7 \mathrm{mg} / \mathrm{l}$. The environmental factors (climate, physico-chemistry characteristics of the water and exogenic inputs, particularly from Oued Beht) seem to control the succession of phytoplankton in El Kansera lake-reservoir. Zooplankton do not regulate algal production (particularly of Microcystis), by reason of the large size of this species and its toxicity. According to an OECD model (1982), El Kansera lake-reservoir is hypereutrophic in 1995 and becomes oligo-mesotrophic in 1996, with a tendency to eutrophication during the summer.

Keywords. - phytoplankton, Cyanobacteria, Diatoms, biomass, chlorophyll, zooplankton, lake-reservoir, eutrophication, Morocco.

\section{INTRODUCTION}

Le développement des populations algales est régi par des facteurs chimiques, biologiques (prédation, compétition...) et physiques (Reynoldes 1984, Sommer 1989). Ces différents paramètres jouent un rôle plus ou moins décisif suivant les périodes de l'année, ce qui entraine des fluctuations dans la composition et l'abondance de la flore algale. La bonne gestion d'un lac-réservoir nécessite donc, entre autres, la connaissance de l'évolution saisonnière du phytoplancton (Le Cohu et al. 1990); celle-ci a été étudiée dans la retenue de barrage El Kansera (Nord-Ouest du Maroc), parallèlement à d'autres approches (Derraz 1995, Fqih Berrada et al. 1998, Fqih Berrada et al. 1999, Fqih Berrada et al. 2000). Dans ce travail, sont pré- sentées et analysées les variations saisonnières de la densité et de la biomasse algale (en poids frais et en chlorophylle a) du phytoplancton global et de deux espèces dominantes; ceci en relation avec certains paramètres abiotiques comme le climat, la disponibilité des nutriments et les apports allochtones de l'oued Beht situé en amont de la retenue, ou encore biotiques (en particulier le zooplancton). Dans la conclusion, ces résultats sont utilisés pour la détermination de l'état trophique de la retenue durant la période d'étude.

\section{MATÉRIEL ET MÉTHODES}

Le lac-réservoir El Kansera (photo 1), l'un des plus anciens du Maroc, se situe au Nord-Ouest du pays, dans le bassin Beht à $40 \mathrm{Km}$ de la ville de 


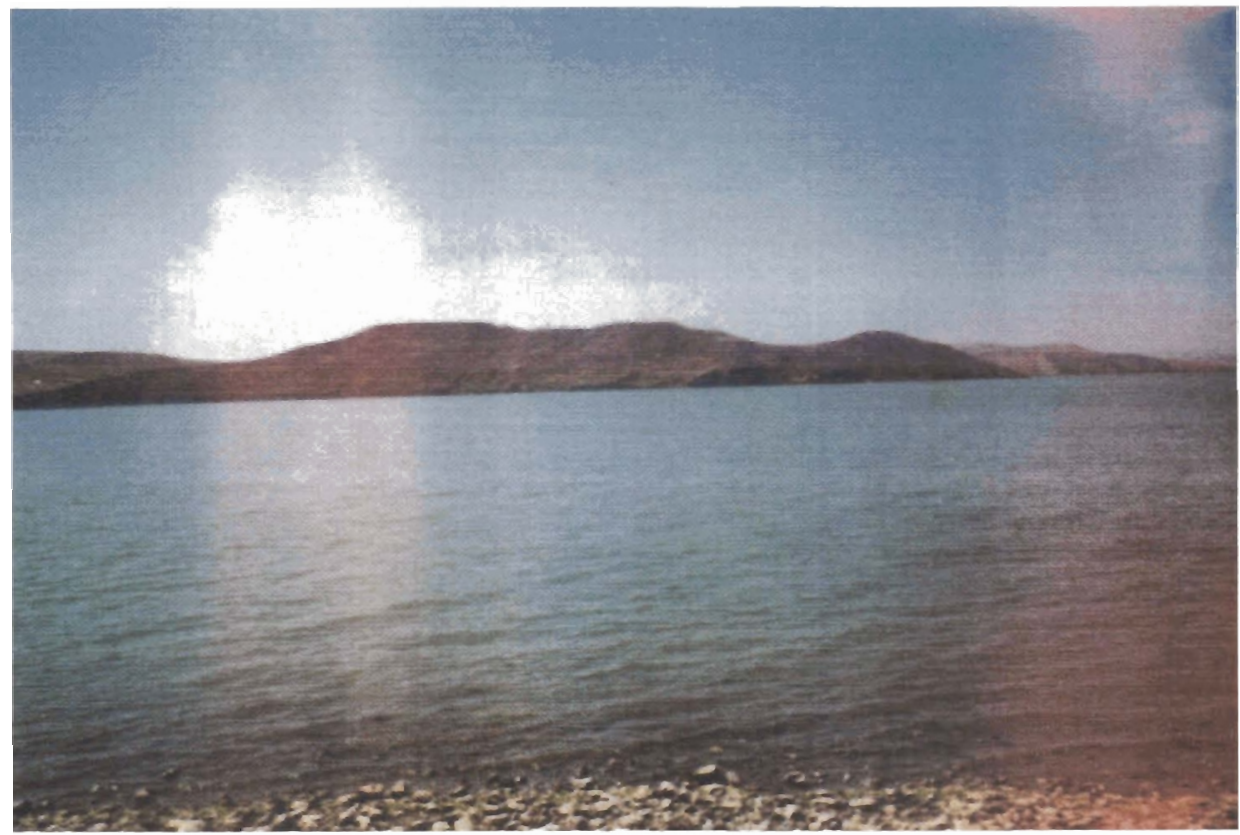

Photo 1 - Photographie du lac-réservoir El Kansera.

Photo 1 - Photography of El Kansera lake-reservoir.

Méknès (fig. 1). Les principales caractéristiques de la retenue sont consignées dans le tableau I. Des prélèvements mensuels ont été effectués entre avril 1995 et août 1996, au niveau de la zone la plus profonde de la retenue $(23 \mathrm{~m})$. Différents niveaux de la colonne d'eau ont été prospectés $(0,5-2-6-10-15$ et $20 \mathrm{~m})$. Les prélèvement ont été réalisés à l'aide d'une colonne verticale de type Van Dorn de capacité de 5 litres. La fixation des algues a été effectuée au formol neutre à $5 \%$ puis au lugol. Le comptage des algues a été réalisé au microscope inversé (méthode de Legendre et al. 1971). La biomasse est exprimée d'une part, en chlorophylle a (méthode de Lorenzen 1967) et d'autre part, en poids frais déterminé après estimation des biovolumes cellulaires (méthode de Lohmann 1908). Les mesures des biovolumes, faites par l'auteur, ont été réalisées à l'aide d'un micromètre oculaire, aprés avoir ramené la forme de l'algue à une forme géométrique connue (tab. II). Pour l'espèce coloniale Microcystis aeruginosa, le comptage et le biovolume ont été évalués à partir des colonies. Les analyses physico-chimiques de l'eau ont été effectuées selon les normes en vigueur, au Laboratoire Public d'Essais et d'Etudes de Casablanca, agréé par le Comité scientifique français d'accréditation 

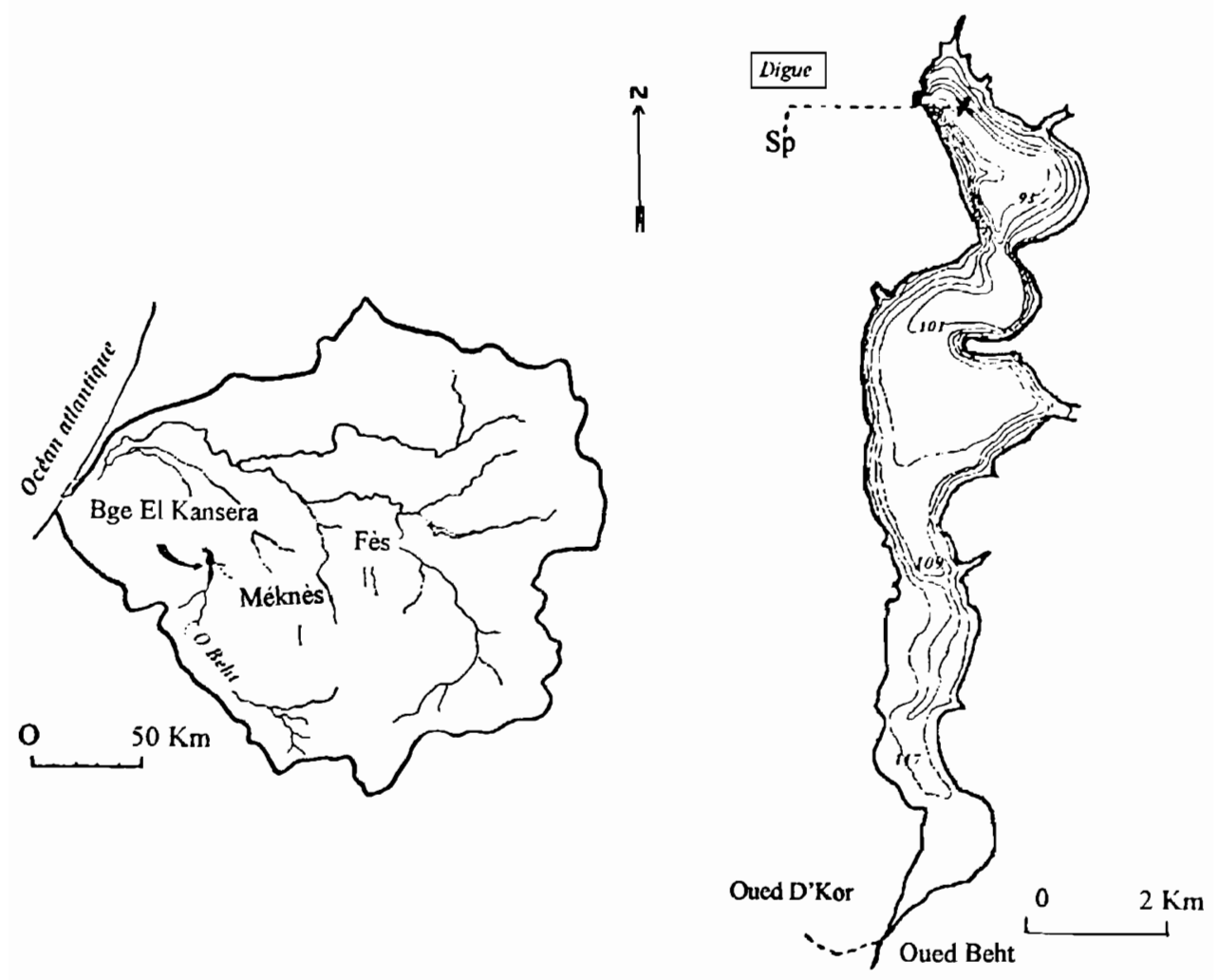

Fig. 1. - Situation géographique et bathymétrie du lac-réservoir El Kansera (d'après Derraz 1995).

Fig. 1. - Geographic location and bathymetry of El Kansera lake-reservoir (from Derraz 1995).

(COFRAC). La transparence et la zone euphotique ont été évaluées à partir de la disparition du Disque de Secchi (AFNOR 1994). Les nitrates et les ions ammoniums ont été dosés par colorimétrie, respectivement par réduction sur colonne de Cadmium en nitrites et au bleu d'indophénol (AFNOR 1994). Le phosphore total a été dosé sur l'eau brute aprés minéralisation en milieu acide, et la silice en présence de molybdate d'ammo- nium (AFNOR 1994). Le comptage du zooplancton a été effectué sur cuve de Dolfuss, soit sur la totalité de l'échantillon, soit sur un sous-échantillon (en fonction de la concentration de ce dernier). Le fractionneur utilisé est la boîte de Motoda, dont l'erreur de fractionnement est de $8,5 \%$ (Pont 1983). Après homogénéisation de l'échantillon fixé au formol, celui-ci est versé sur toute la surface de la cuve constituée de 200 cases. 
Tableau I. - Caractéristiques morphométriques du lac-réservoir El Kansera (d'aprés Derraz, 1995 et la Direction Nationale de Météorologie du Maroc).

Table I. - Morphometric characteristics of the El Kansera lake-reservoir (from Derraz, 1995 and the National Direction of Meteorology of Morocco).

Année de mise en eau

Superficie du bassin versant

Province

Fonction

Type

Hauteur sur fondation

Longueur en crête

Superficie du plan d'eau

Longueur moyenne

Durant la période 1995-1996

$\begin{array}{lll}\text { Variations de: } & \text { volume de la retenue }\left(\mathrm{Mm}^{3} / \mathrm{mois}\right) & (67-267) \\ & \text { volume des apports }\left(\mathrm{Mm}^{3} / \mathrm{mois}\right) & (5-200) \\ & \text { restitution }\left(\mathrm{Mm}^{3} / \mathrm{mois}\right) & (2-40) \\ & \text { temps de séjour de l'eau (mois) } & (1,7-22) \\ \text { profondeur maximale }(\mathrm{m}) & (5-23)\end{array}$

1935

$4500 \mathrm{~km}^{2}$

Sidi Slimane

Energie électrique

Eau Potable, Irrigation

Béton poids et contreforts

$68 \mathrm{~m}$

$170 \mathrm{~m}$

$18 \mathrm{~km}^{2}$

$14 \mathrm{Km}$

$(\min -\max )$

Tableau II. - Valeurs moyennes des biovolumes calculés par l'auteur de quelques espèces algales retrouvées dans le lac-réservoir EI Kansera.

Table II. - Mean values of biovolume (calculated by the author) of a few algal species in El Kansera lake-reservoir.

espèces algales

Microcystis aeruginosa

Strombomonas sp.

Cyclotella ocellata

Crucigenia apiculata

Scenedesmus ecornis

Oocystis lacustris

Oocystis crassa biovolume moyen / cellule ou colonie $\left(\mu \mathrm{m}^{3}\right)$

$65.410 .000\left({ }^{*}\right)$

58

1300

58

1050

768

1223

- Biovolume colonial moyen. 


\section{RÉSULTATS ET DISCUSSION}

\subsection{Environnement abiotique}

\subsubsection{Niveau du plan d'eau, transparence et zone euphotique}

Les faibles précipitations enregistrées en 1995 (année sèche) se traduisent par une diminution accusée des apports hydriques (fig. 2A) et par conséquent du volume de la retenue; ce qui induit une nette diminution du niveau du plan d'eau (minimum : $5 \mathrm{~m}$, fig. 2B). A l'inverse, en 1996 (année pluvieuse), la forte pluviométrie et les crues de l'oued Beht entraînent une élévation sensible du niveau du plan d'eau (maximum : $23 \mathrm{~m}$ ).

La zone euphotique (fig. 2B) déterminée à partir de la transparence de l'eau (Reynolds 1984), oscille entre un minimum de $0,4 \mathrm{~m}$ (été-automne 1995) et un maximum de $7,7 \mathrm{~m}$ (printemps 1996). Cette augmentation sensible de la zone euphotique en 1996 est synchrone avec l'élévation du niveau du plan d'eau de la retenue.

\subsubsection{Température, $\mathrm{pH}$ et Oxygène dissous}

Le lac-réservoir EI Kansera est un lac monomictique chaud, caractérisé par une seule période de mélange automno-hivernale et une période de stratification thermique estivale; celleci débute dés le mois d'avril et prend fin au mois de septembre (fig. 3). L'écart maximal entre la surface et le fond atteint $13,1^{\circ} \mathrm{C}$ et est enregistré en juin 1996, en raison du meilleur remplissage de la retenue durant cette année. Cet écart thermique se rapproche de celui d'autres retenues marocaines (Abouzaid 1986, Derraz 1987), mais est plus élevé que celui observé dans certains lacs situés sous climat tempéré (Capblancq 1974) ou chaud (lac Victoria an niveau de l'équateur, cité par Derraz 1987).

Le $\mathrm{pH}$ des eaux est alcalin et varie entre 7 et 8,7 (fig. 4). Les valeurs maximales sont observées aux saisons printano-estivales, dans les eaux de surface où l'activité photosynthétique est plus intense (mesures non faites). La période de brassage induit une homogénéisation et une baisse du $\mathrm{pH}$ sur toute la colonne d'eau.

L'évolution saisonnière de l'oxygène dissous, exprimé en pourcentage de saturation (fig. 5), indique une sursaturation des couches superficielles en période de printemps et été de chaque année, avec un pic de $138 \%$ en juin 1995. Ce maximum serait lié à une intense activité photosynthétique dans les eaux de surface. Parallèlement, les eaux du fond subissent une désoxygénation progressive, aboutissant à l'installation d'une couche quasi-anoxique avec un minimum de $3,2 \%$ en juillet 1996 . Cette désoxygénation serait le résultat d'une intense activité bactérienne dans les eaux du fond (mesures non faites). Un tel profil est caractéristique des lacs eutrophes (Wetzel 1983). La période de brassage entraîne une réoxygénation plus ou moins complète de toute la masse d'eau. 

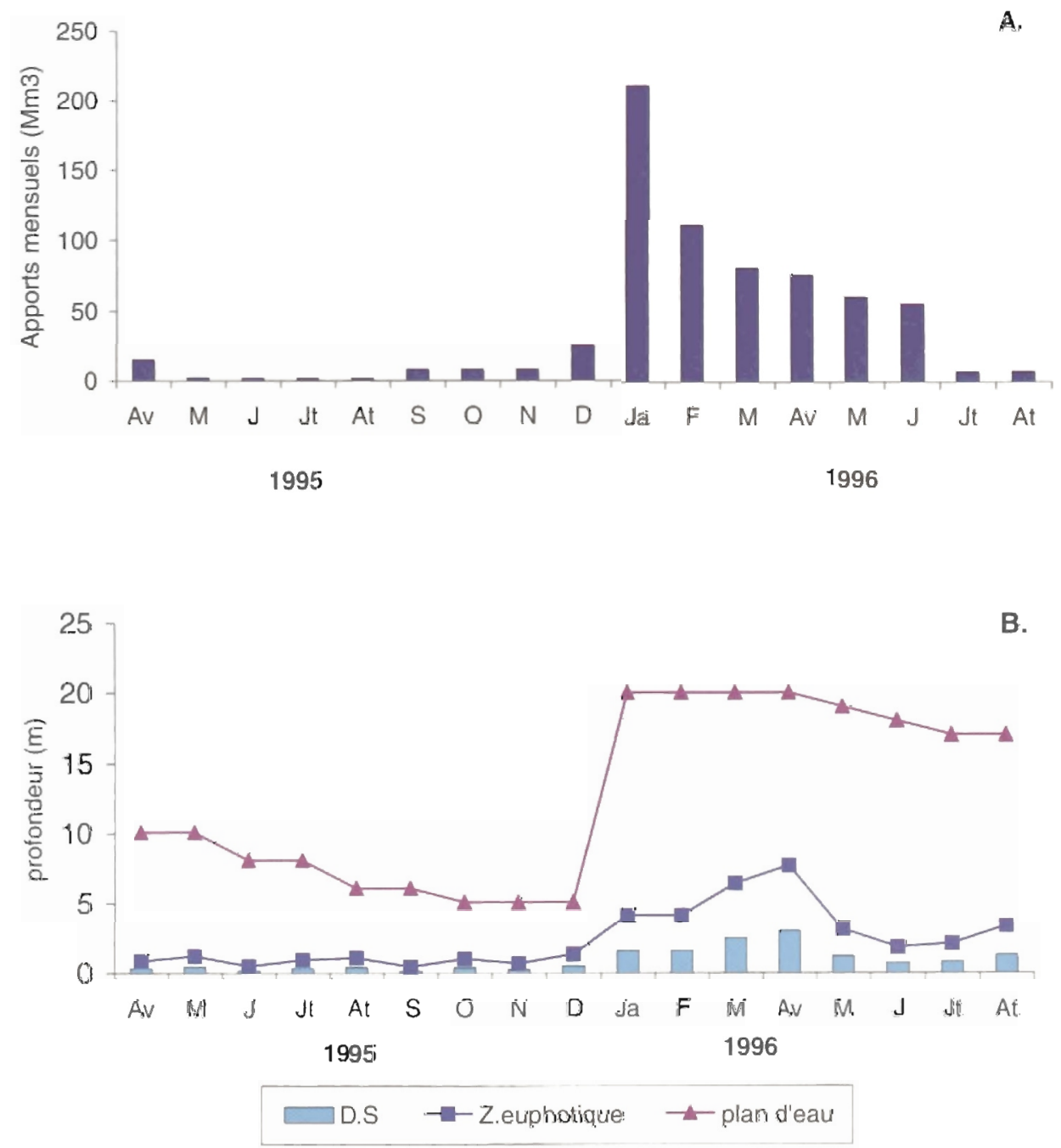

Fig. 2. - Variations saisonnières des apports à la retenue El Kansera (A) et variations saisonnières du niveau du plan d'eau, de la transparence de l'eau (Disque de Secchi, D.S) et de la zone euphotique dans cette retenue $(B)$.

Fig. 2. - Seasonal variations in inputs in El Kansera lake-reservoir (A) and seasonal variations in water level, transparency (Secchi Disc, D.S) and euphotic zone in this lake-reservoir (B).

Fig. 3. - Profils verticaux de la température dans la retenue El Kansera, durant la période d'étude. Fig. 3. - Vertical temperature profiles in El Kansera lake-reservoir during the study period. 

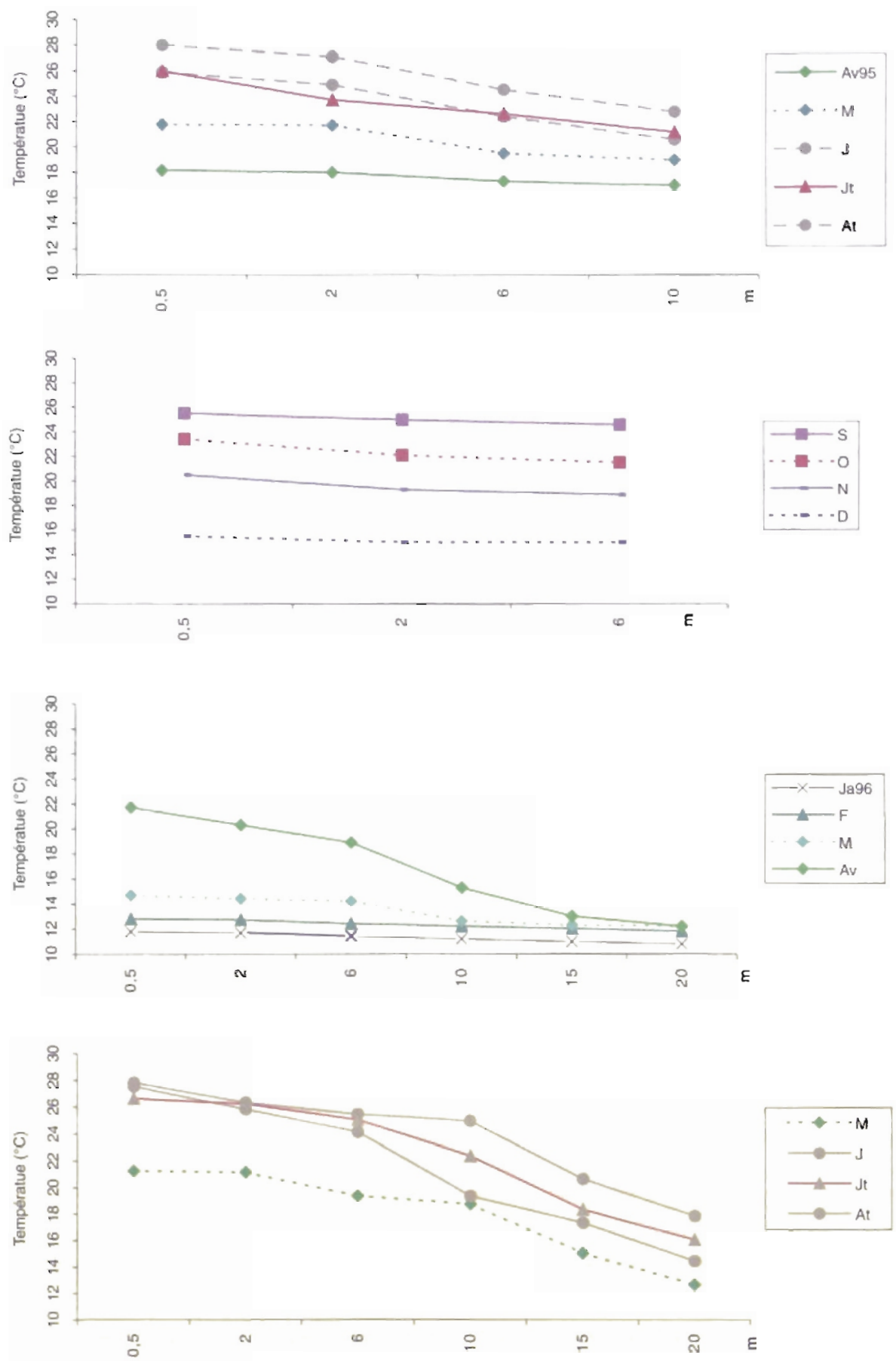


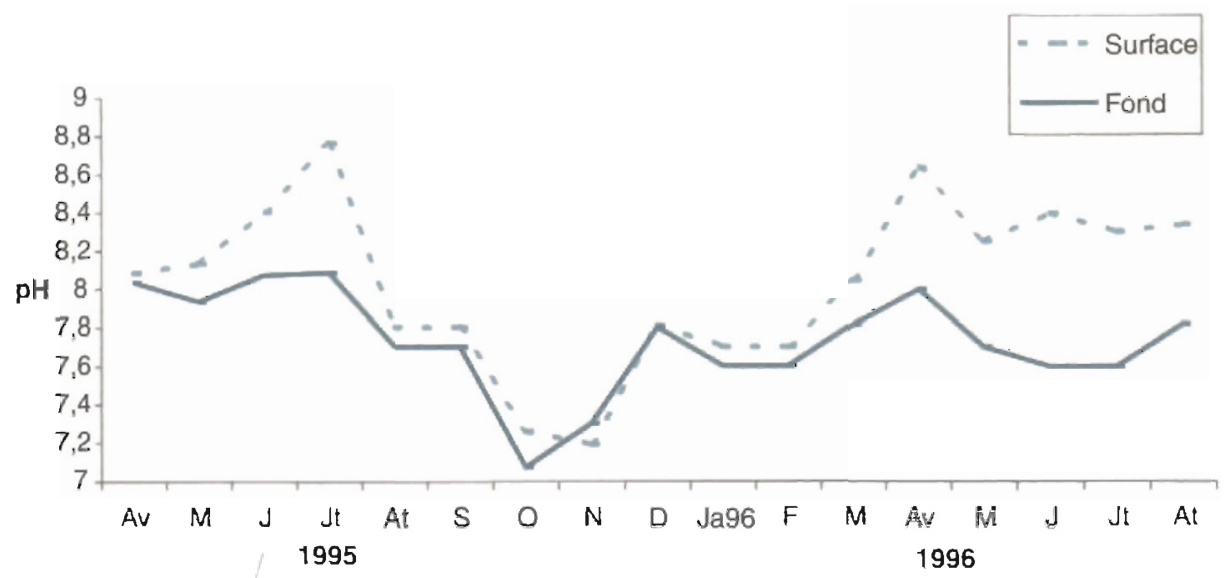

Fig. 4. - Variations saisonnières du pH en surface et au fond de la retenue durant la période d'étude.

Fig. 4. - Seasonal variations in $\mathrm{pH}$ at the surface and boitom of the lake-reservoir during the study period.

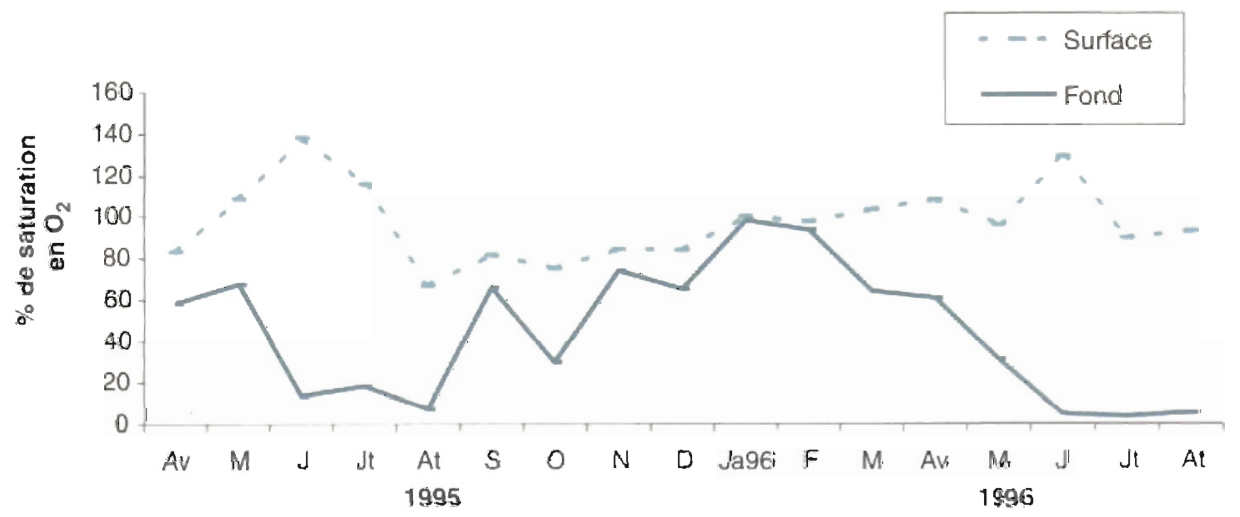

Fig. 5. - Variations saisonnières du pourcentage de saturation en oxygène en surface et au fond de la retenue durant la période d'étude.

Fig. 5. - Seasonal variations in the oxygen saturation rate at the surface and bottom of the lake-reservoir during the study period.

\subsubsection{Sels nutritifs}

Les teneurs en nutriments azotés et phosphorés présentent des fluc- tuations importantes dans la retenue. Les concentrations en nitrates $\left(\mathrm{NO}_{3}\right)$, constituant les fortes proportions de l'azote total, varient avec les saisons 
et les niveaux de la colonne d'eau (fig. 6A). En été 1995, la diminution sensible des nitrates dans les eaux de surface coïncide avec le développement des blooms à Cyanobactéries (voir chapitre 3-2), alors que les faibles concentrations dans les eaux du fond sont dues à leur réduction. L'apparition synchrone d'un pic d'ions ammoniums, de l'ordre de $854 \mu \mathrm{g} / \mathrm{l}$ (fig. 6B), marque cette réduction dans l'hypolimnion désoxygéné. Parallèlement, un léger enrichissement des eaux en phosphore total $(125 \mu \mathrm{g} / \mathrm{l}$ de PT) et en orthophosphates $\left(25 \mu \mathrm{g} / \mathrm{l}\right.$ de $\left.\mathrm{PO}_{4}\right)$ est enregistré (figs. $6 \mathrm{C}$ et $6 \mathrm{D}$ ). En réalité, la faible profondeur de la colonne d'eau durant cette année sèche, associée aux vents quasi-permanents qui règnent au niveau de la retenue (Chergui), favoriseraient largement la resuspension des sédiments fortement enrichis en phosphore (Piersen \& Weyhenmeyer 1994, Derraz 1995). Durant la saison automnale (octobre), les pics de phosphore $\left(155 \mu \mathrm{g} / \mathrm{l}\right.$ de PT et $95 \mu \mathrm{g} / /$ de $\mathrm{PO}_{4}$ ) trouvent leur origine dans la resuspension des sédiments par brassage des eaux de niveaux peu profonds (phase d'homogénéisation thermique). En revanche, les concentrations en nitrates demeurent peu élevées (3,9 mg/l). En réalité, durant cette année sèche, les valeurs relativement faibles du rapport $\mathrm{N}-\mathrm{NO}_{3} / \mathrm{P}$ $\mathrm{PO}_{4}$ (fig. 7) indiquent le rôle limitant de l'azote dans le lac-réservoir. L'année suivante, ce rapport s'inverse : le lessivage du bassin versant provoqué par les pluies torrentielles et les crues de l'oued Beht (figs. 6A, $6 C$ et $6 D$ ) déversent une quantité importante de nutriments azotés et phosphorés dans le lac; les teneurs en nitrates atteignent un maximum de 23,1 mg/l en mai, tandis que celles du phosphore restent relativement faibles la majeure partie de l'année. En réalité, tout au long de l'année, des quantités importantes de phosphore sont stockées dans le sédiment par précipitation sous forme d'hydroxyapatite et adsorption sur les particules en suspension (Abdallaoui et al. 1998). Mais en été, l'anoxie qui règne au fond de la retenue entraîne la réduction du phosphore et sa libération dans la colonne d'eau. C'est le phénomène de relargage, à l'origine du pic de PT $(156 \mu \mathrm{g} / \mathrm{l})$ et de $\mathrm{PO}_{4}$ $(95 \mu \mathrm{g} / \mathrm{l})$ en cette saison.

Les teneurs en silice qui oscillent entre un minimum de $6 \mathrm{mg} / \mathrm{l}$ et un maximum de $11,5 \mathrm{mg} / \mathrm{l}$ (fig. 8 ), ne montrent pas de variations saisonnières importantes; cet élément comparé

Fig. 6. - Variations saisonnieres des teneurs en nitrates (A), en ions ammoniums (B), en phosphore total (C) et en orthophosphates (D) en surface et au fond de la retenue, et dans l'oued Beht ( $A, C$ et D).

Fig. 6. - Seasonal variations in nitrates (A), ammonium ions (B). total phosphorus (C). and of dissolved phosphorus PO: (D) at the surface and bottom of the lake-reservoir. and in the Beht Oued (A, C and D). 
A.

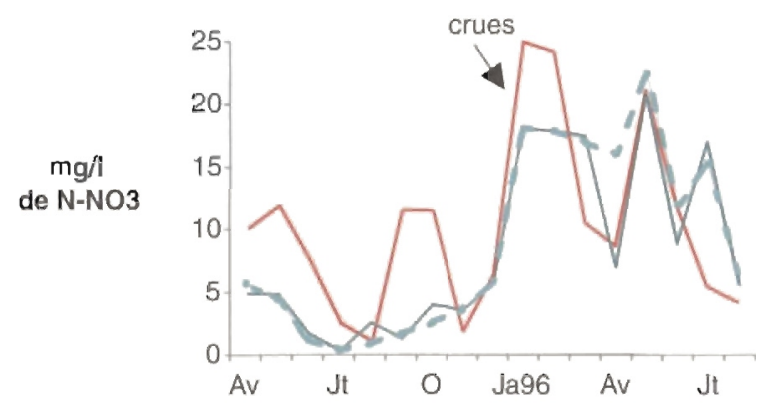

- Surface

- Fond

O.Beht

B.

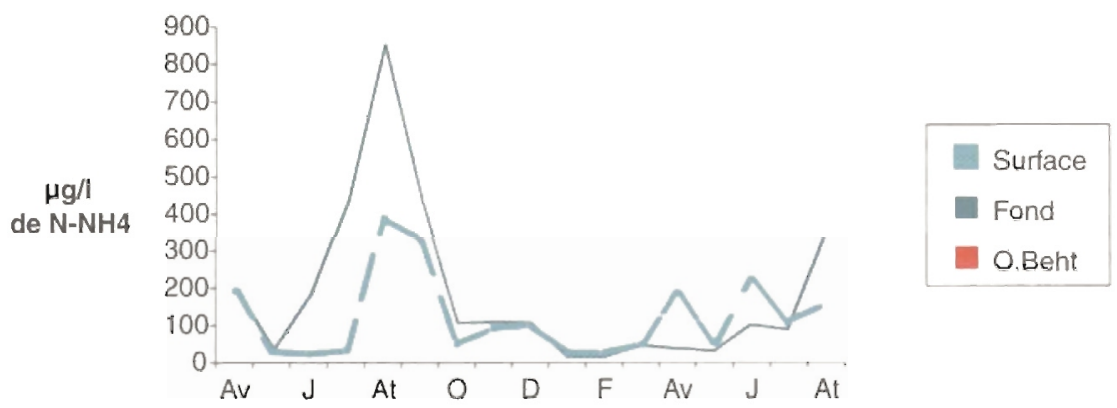

c.

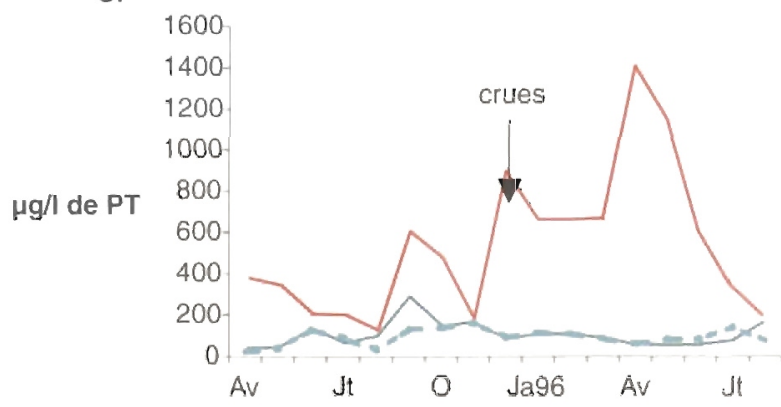

Surface

Fond

O.Beht

D.

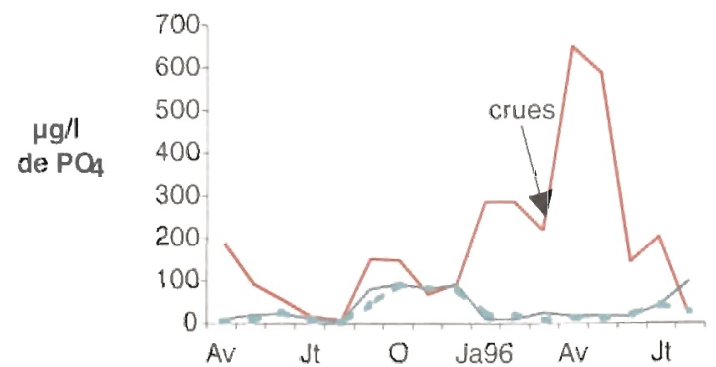

Surface

Fond

O.Beht 


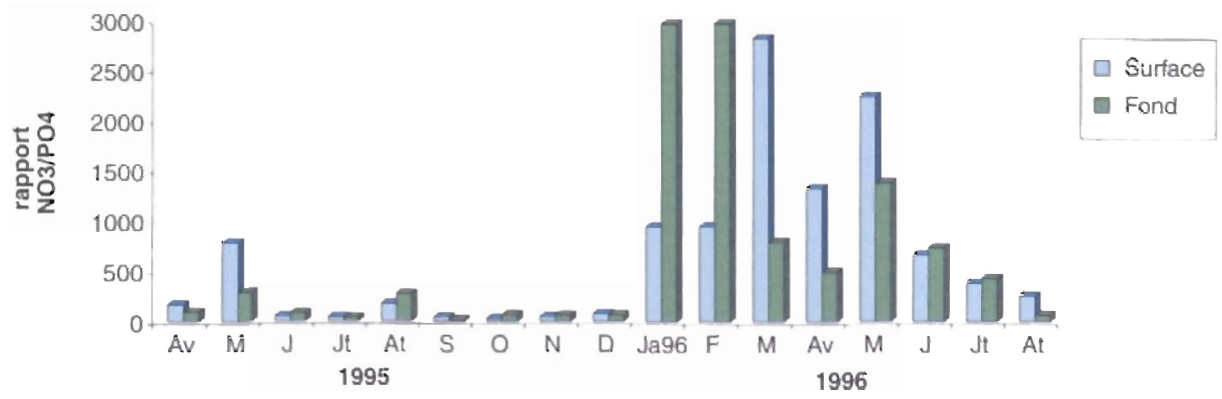

Fig. 7. - Variations saisonnières du rapport N-NO $/ \mathrm{P}_{3}-\mathrm{PO}_{4}$ en surface et au fond de la retenue durant la période d'étude.

Fig. 7. - Seasonal variations in the N-NO $/ \mathrm{P}-\mathrm{PO}_{4}$ ratio at the surface and bottom of the lake-reservoir during the study period.

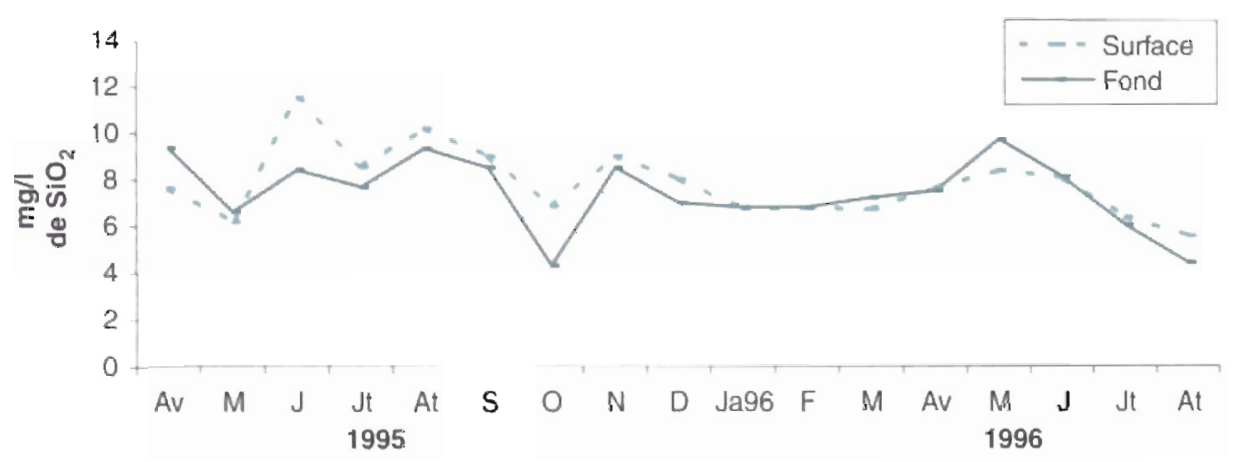

Fig. 8. - Variations saisonnières de la silice en surface et au fond de la retenue durant la période d'étude.

Fig. 8. - Seasonal variations of silica $\mathrm{SiO}_{2}$ at the surface and bottom of the lake-reservoir during the study period.

à d'autres lacs-réservoirs marocains (Loudiki 1990), ne constituerait pas un facteur limitant pour le développement des algues (Diatomées) dans la retenue.

\subsection{Evolution saisonnière du phytoplancton global}

3.2.1 Variations saisonnières de la biomasse algale

L'évolution de la biomasse algale dans la zone euphotique (fig. 9) 


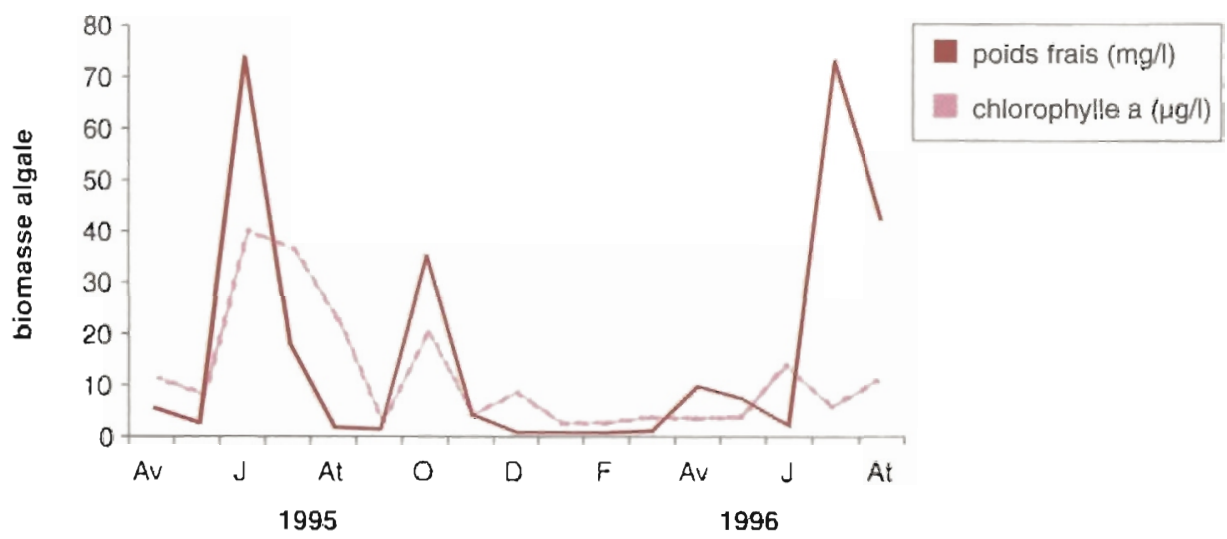

Fig. 9. - Variations saisonnières de la biomasse algale en poids frais $(\mathrm{mg} / \mathrm{l})$ et en chlorophylle a $(\mu g / l)$ dans la zone euphotique de la retenue.

Fig. 9. - Seasonal variations in the algal biomass in wet weight (mg/l) and in chlorophyll a $(\mu g / l)$ in the euphotic zone of the lake-reservoir.

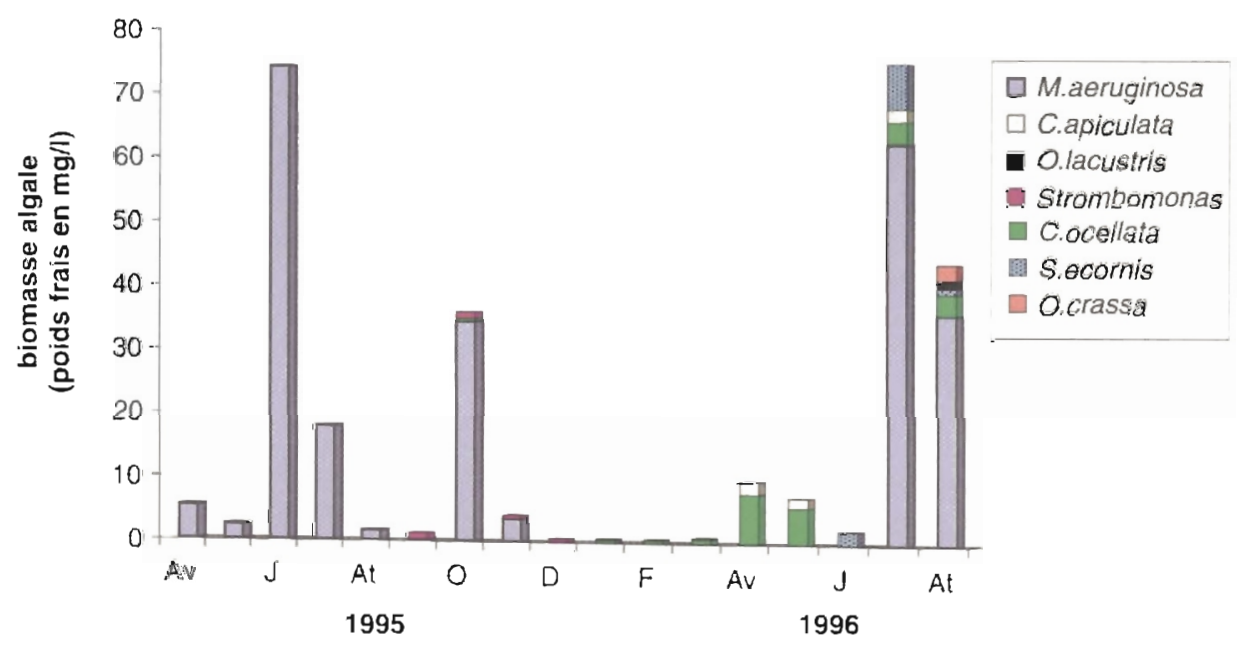

Fig. 10. - Successions temporelles des principales espèces algales (exprimées en poids frais) dans la zone euphotique de la retenue durant la période d'étude.

Fig. 10. - Successive colonization by the principal algal species of the euphotic zone of the lake-reservoir during the siudy period (in wet weight).

indique d'importantes variations temporelles. L'année 1995 est caractérisée par deux poussées algales (73 mg/l en juin et $34 \mathrm{mg} / \mathrm{l}$ en oc- tobre). Elles sont principalement liées au développement de la Cyanobactérie Microcystis aeruginosa (fig. 10). Le biovolume très important de cette 
espèce coloniale (tab. II) est à l'origine des fortes valeurs de biomasse (poids frais). Les conditions de sécheresse du milieu (températures élevées de l'eau, grande stabilité du milieu due en partie aux faibles apports allochtones et faibles teneurs en nutriments) semblent favoriser le développement de cette espèce, dotée d'une grande capacité d'adaptation. La saison hivernale est marquée par un déclin du phytoplancton; les turbulences hydrodynamiques violentes liées aux crues de l'oued Beht et aux lâchés d'eau, associées aux basses températures de la saison (fig. 3), expliqueraient cette situation. Dés le printemps suivant (avril), une nouvelle poussée algale apparaît $(9,4 \mathrm{mg} / \mathrm{l})$ avec des espèces de petites tailles; Cyclotella ocellata et Crucigenia apiculata (fig. 10).
L'augmentation de la température printanière (fig. 3) et l'enrichissement du milieu en sels nutritifs (figs. 6A, 6C et 6D) après les fortes pluies hivernales, stimuleraient ce développement algal. De plus, l'augmentation de la transparence de l'eau en cette saison (fig. 2) favoriserait le développement de Cyclotella notamment, à caractère héliophile reconnu (Loudiki 1990). En juin, la biomasse algale baisse à nouveau ( $2 \mathrm{mg} / \mathrm{l}$ ). La disparition de $\mathrm{Cy}$ clotella et de Crucigenia en cette période coïncide avec la prolifération du zooplancion herbivore dans le lacréservoir (fig. 11); en effet, un pic d'espèces herbivores est enregistré en cette période et est constitué de Cladocères (Diaphanosoma brachyurum), de Rotifères (Polyarthra vulgaris et Gastropus hyptopus) et de Copépodes (Acanthocyclops robus-

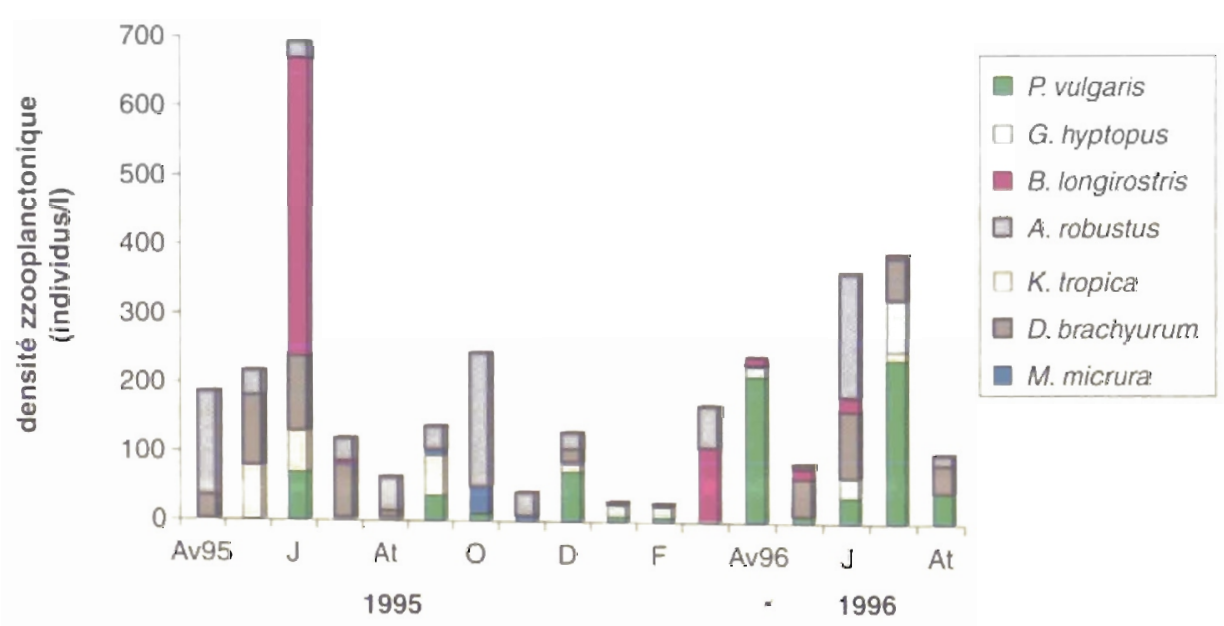

Fig. 11. - Évolution saisonnière du zooplancton total et des principales espèces herbivores dans la retenue (valeurs moyennes de la colonne d'eau).

Fig. 11. - Seasonal evolution in total zooplankton and the principal herbivorous species in the lake reservoir (mean values in the water column). 
tus aux stades juvéniles). Seule l'algue Scenedesmus ecornis se développe plus ou moins bien en présence de ce zooplancton. Des expériences de Moncef (1993) ont en effet montré que la Diatomée Cyclotella en particulier subissait la plus forte activité de broutage en présence d'autres algues de petites tailles du genre Scenedesmus. Le mois suivant, une nouvelle poussée algale est enregistrée $(72,7 \mathrm{mg} / \mathrm{l})$ et est imputable à la Cyanobactérie Microcystis aeruginosa qui recolonise le milieu. Le relargage du phosphore en cette saison (figs. $6 \mathrm{C}$ et $6 \mathrm{D}$ ) stimulerait en bonne partie la réapparition de cette algue.

\subsubsection{Variations saisonnières de la chlorophylle a}

L'évolution saisonnière de la chlorophylle a dans la zone euphotique (fig. 9) suit plus ou moins bien celle de la biomasse algale. Une corrélation assez élevée $(r=0,6)$ a été trouvée entre ces deux paramètres. Les pics printano-estivaux de chlorophylle coincident souvent avec les blooms algaux (par exemple, le pic de $40 \mu \mathrm{g} / \mathrm{l}$ de chlorophylle en juin 1995 coïncide avec celui de Microcystis aeruginosa). A l'inverse, les minima de chlorophylle correspondent au déclin de la communauté algale (en hiver notamment). Toutefois, certaines discordances apparaissent, en particulier durant l'année 1996: au printemps par exemple (avril), de faibles teneurs en chlorophylle sont enregistrées $(3,2 \mu \mathrm{g} / \mathrm{l})$, en dépit d'une poussée algale importante. Cette dis- cordance semble être liée aux variations de la teneur cellulaire en chlorophylle qui dépend notamment de la densité cellulaire (Holmes 1966, Devaux et al. 1982), des quotas cellulaires en nutriments (Dauta 1982, Capblancq 1982) et des conditions d'éclairement (un éclairement intense entraîne une réduction du contenu cellulaire en chlorophylle, Testard 1983). Une situation analogue a été observée dans la retenue Hassan ler au Sud du Maroc (Loudiki 1990). En juin, la situation est inverse : un pic de chlorophylle a est enregistré $(13,5 \mu \mathrm{g} / \mathrm{l})$, au moment où la biomasse algale baisse sensiblement. Ceci semble être en relation avec la diminution brutale de la transparence de l'eau (fig. 2), qui limite le taux de pénétration de la lumière dans la colonne d'eau. Ce qui induit les cellules algales à une synthèse accrue de la chlorophylle pour capter, au mieux, l'énergie solaire (Testard 1983, Loudiki 1990).

\subsection{Evolution spatio-temporelle de deux espèces dominantes}

\subsubsection{Population de Microcystis aeruginosa KUTZ}

La Cyanobactérie Microcystis aeruginosa (Kutz.) est une espèce autochtone dans la retenue El Kansera, Gayral-Engerbaud l'ayant déjà signalée dans le lac-réservoir depuis 1954. Durant l'année 1995, elle se développe de façon précoce et présente un bloom très important dès le mois de juin. A ce moment de l'année, 
l'espèce se cantonne dans les couches superficielles $(-0,5$ à $-2 \mathrm{~m})$, avec en moyenne 764 à 927 colonies/l (fig. 12). La concentration de Microcystis dans les couches de surface est favorisée par son pouvoir de flottabilité grâce à la présence de pseudo-vacuoles (Zohary \& Robart 1989, Konopka et al. 1993), qui lui permettent de se maintenir dans les couches supérieures les plus chaudes $\left(25^{\circ} \mathrm{C}\right)$ et les plus accessibles à la lumière (la faible transparence de l'eau réduit la pénétration de la lumière dans la colonne d'eau). Ces observations confirment les résultats de Varis (1993) qui a observé des blooms de la Cyanobactérie dans des lacs eutrophes et turbides, à des températures variant de 19 à $25^{\circ} \mathrm{C}$. Par ailleurs, les teneurs en sels nutritifs en cette période montrent un épuisement des nitrates sur toute la colonne d'eau, en même temps qu'un léger enrichissement en éléments phosphorés. Lewis (1983) explique la formation des blooms à Microcystis par la grande adaptation de cette algue à la limitation des eaux en azote. II apparaît de plus, que cette espèce est très compétitive sur l'élément phosphore par rapport aux autres Cyanobactéries (Oscillatoria, Phormidium, Lyngbya...); elle serait la mieux adaptée pour l'assimilation et le stockage du phosphore. Selon Sbiyyaa et al. (1997), cette algue est dotée d'une grande capacité de stockage intracel- lulaire de l'azote et du phosphore. De plus, ces blooms sont rarement broutés par le zooplancton en général, en raison du caractère toxique de la Cyanobactérie (Zhen-rong 1992, Thébault et al. 1995, Enrisken \& Moestryp 1997), et de la grande taille des colonies qui auraient tendance à colmater l'appareil de filtration des Crustacés microfiltreurs (Porter \& Orcutt 1980). C'est ainsi qu'au mois de juin, le pic zooplanctonique constitué d'espèces herbivores telles Bosmina longirostris, Diaphanosoma brachyurum, Polyarthra vulgaris et Keratella tropica coïncide avec le bloom de Microcystis (fig. 11); ce qui indiquerait la faible pression de broutage de ce zooplancton herbivore vis-à-vis de cette espèce. Parallèlement, et sous l'action des vents de direction Est (Chergui), les colonies "flottantes" de Microcystis forment un tapis étalé sur plusieurs hectares vers l'amont de la retenue (Fqih Berrada et al. 2000). Les blooms à Microcystis ont déjà été observés dans la retenue El Kansera en 1991-1992 (Derraz 1995) et dans d'autres retenues marocaines comme le Lalla Takerkoust (Cherifi 1992, Loudiki et al. 1995), ainsi que dans plusieurs lacs-réservoirs situés sous des latitudes différentes comme au Japon dans le lac Suwa (Watanabe \& Oish 1983), ou en Afrique du Sud dans le réservoir Hartbeesport (Zohary \& Robarts 1989). Au mois de juillet,

Fig. 12. - Répartition verticale de Microcystis aeruginosa (colonies/l) dans la retenue.

Fig. 12. - Vertical distribution of Microcystis aeruginosa (colonies/l) in the lake-reservoir. 

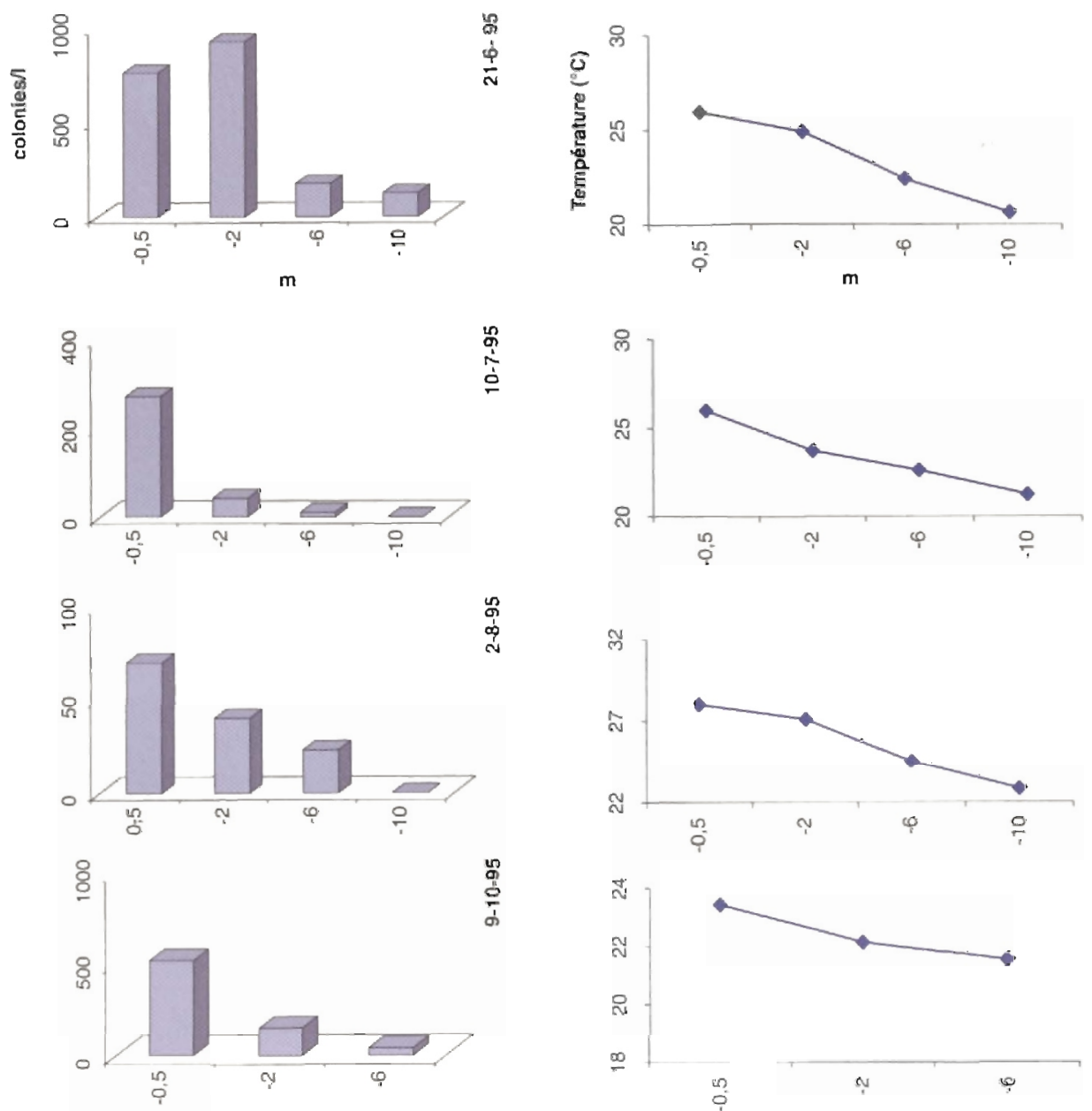

๕ั
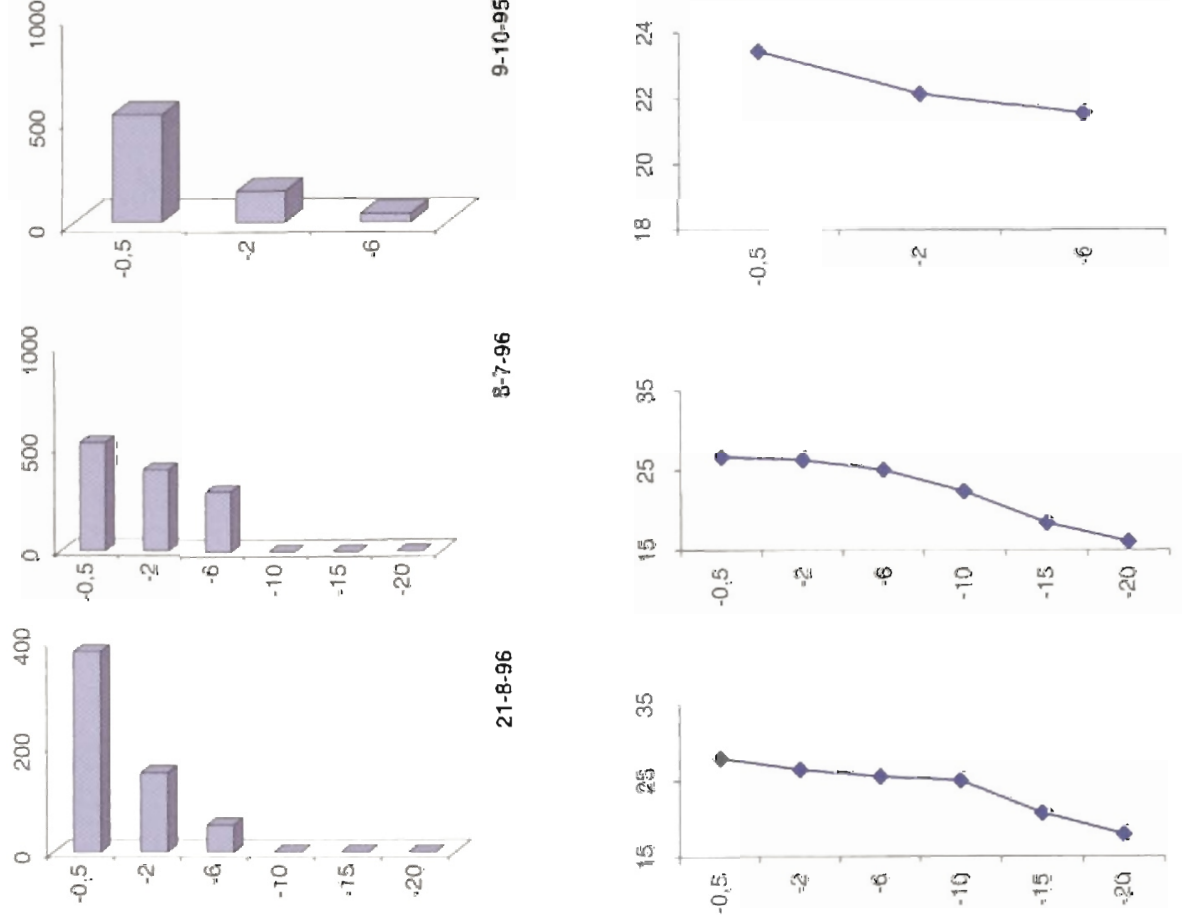
la baisse sensible de la biomasse de Microcystis dans la colonne d'eau n'est pas réelle; elle serait liée, en effet, au pouvoir de flottabilité de l'espèce (Zohary \& Robarts 1989), qui lui permet d'être facilement entraînée par les vents vers des zones plus calmes de la retenue.

Durant la saison automnale (octobre), un nouveau bloom de la Cyanobactérie est enregistré. Malgré l'homogénéité thermique qui caractérise les eaux de la retenue en cette période $\left(22^{\circ} \mathrm{C}\right.$ sur toute la colonne d'eau), les colonies se maintiennent en surface $(-0,5 \mathrm{~m})$ avec environ 520 colonies/l. Le maintien de Microcystis à la surface de l'eau en période de brassage serait lié à son pouvoir de flottabilité, favorisé par les pseudovacuoles. Ce bloom algal coïncide avec une augmentation sensible des teneurs en phosphore, en rapport avec le brassage automnal. Ce qui rejoint les observations de Köhler (1992), de Varis (1993) et de Derraz (1995) qui montrent l'importance du phosphore sur le développement de cette espèce.

L'année suivante, Microcystis aeruginosa recolonise le milieu plus tardivement, en juillet. En cette période, les colonies se concentrent dans les couches superficielles entre $-0,5$ et $-6 \mathrm{~m}$ et atteignent une densité de 292 à 528 colonies/l. Cette période coïncide avec une phase de stratification thermique bien marquée, séparant un épilimnion chaud (25 à $27^{\circ} \mathrm{C}$ ) d'un hypolimnion relativement froid (16 à $18^{\circ} \mathrm{C}$ ). Les teneurs en nitrates montrent des valeurs importantes durant toute l'année (apports allochtones importants). En même temps, un enrichissement du milieu en phosphore est observé durant la saison estivale (phénomène de relargage). Ces observations confirment les expériences de Derraz (1995) qui montrent que, sous des conditions nutritives non limitantes, le taux de croissance maximal de Microcystis isolée de la retenue El Kansera n'est fonction que de l'éclairement et de la température. Ainsi, la valeur la plus élevée du taux de croissance de l'espèce $\left(\mu_{\operatorname{mix}}=\right.$ 1,28 .jour ') a été obtenue à une intensité de $\left(I_{\text {opt }}=220 \mu \mathrm{E} \cdot \mathrm{m}^{2} \cdot \mathrm{s}^{-1}\right)$ et une température de $\left(\mathrm{T}_{\text {opt }}=30^{\circ} \mathrm{C}\right)$ pour une photopériode à cycle lumière/obscurité de 15/9. Zohary \& Robarts (1989) ont noté, pour leur part, un développement intense de cette Cyanobactérie dans le réservoir Hartbeesport (Afrique du Sud), où les nutriments (N et $P$ ) sont disponibles, et aussi bien en période de faibles température et lumière qu'en période estivale.

\subsubsection{Population de Cyclotella ocellata (Pantocksek)}

La Diatomée Cyclotella ocellata est la seconde espèce autochtone dans la retenue El Kansera (Gayral 1954). En 1995, elle semble très af-

Fig. 13. - Répartition verticale de Cyclotella ocellata (cellules/l) dans la retenue.

Fig. 13. - Vertical distribution of Cyclotella ocellata (cells/I) in the lake-reservoir. 

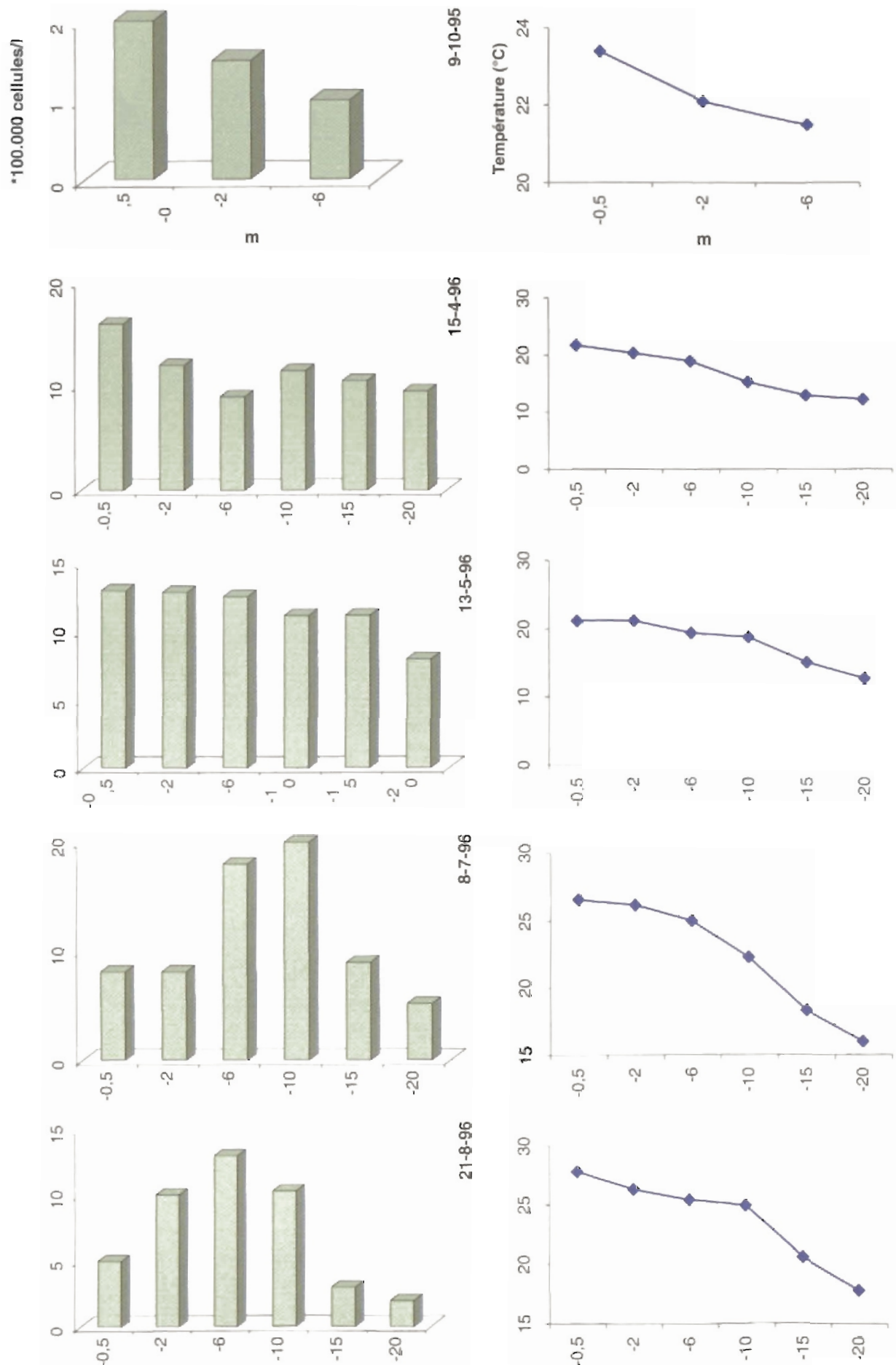
fectée par les conditions de sécheresse, surtout par les températures élevées étalées sur toute l'année et la carence en nitrates. Ainsi en dépit des fortes concentrations en silice (fig. 8), élément majeur pour les Diatomées, Cyclotella ocellata n'apparait qu'en saison automnale avec une densité négligeable (fig. 13). Le broutage zooplanctonique effectué par le Cladocere herbivore Moina micrura et les Rotifères (Polyarthra vulgaris, Gastropus sp...) contribuerait également à cette baisse (fig. 11). En effet, la mauvaise qualité de la nourriture algale en cette période (Cyanobactéries) fait que ce broutage est particulièrement "ciblé " sur cette petite Diatomée d'ingestion facile (Moncef 1993).

L'année suivante, suite à l'enrichissement du milieu en éléments nutritifs (azote et silice), la Diatomée Cyclotella ocellata recolonise le milieu en saison printanière, à des densités très élevées. Elle se développe de façon homogène sur toute la colonne d'eau, avec une légère préférence pour les couches superficielles $(-0,5$ à $-2 \mathrm{~m})$, où les températures varient de 20 à $21,7^{\circ} \mathrm{C}$. Ces observations sont en accord avec les résultats de Capblancq (1972) et de Loudiki (1990), qui notent que les espèces du genre Cyclotella apparaissent comme des formes héliophiles, qui se développent de façon optimale lorsque la température avoisine $20^{\circ} \mathrm{C}$. Cette constatation rejoint également celles faites par de nombreux auteurs (Hermann 1980, Cardinal 1983). Au mois de juin, le déclin de
Cyclotella coïncide avec un nouveau pic zooplanctonique herbivore (fig. 11). Cet effet de broutage est de plus accentué par l'introduction dans le lac de carpillons argentés de Chine à régime herbivore (ONEP 1996). Le déclin de la Diatomée serait également lié à une sédimentation très rapide des frustules (début de la phase de stratification). Dès le mois de juillet, Cyclotella ocellata présente un seconde poussée, parallèlement à une légère augmentation des teneurs en éléments nutritifs (nitrates et silice). La régénération par le broutage zooplanctonique de ces sels nourriciers constituerait un stimulus pour la Diatomée. Ce pic, situé entre -6 et $-10 \mathrm{~m}$ de profondeur, coïncide avec l'installation de la thermocline à ce niveau. II apparaît en effet, que la Diatomée progresse au niveau de la zone euphotique, pour fuir les fortes radiations et les hautes températures estivales (supérieures à $25^{\circ} \mathrm{C}$ ). Ce même phénomène a été observé dans le lac de Pareloup (Jrad 1989) et dans la retenue Hassan ler, au Sud du Maroc (Loudiki 1990). Au mois d'août, la Diatomée persiste sur toute la colonne d'eau, mais en réalité la majorité des cellules observées sont moribondes à cause du phénomène de photooxydation, ou d'une éventuelle altération par les toxines de Microcystis.

Ainsi, la croissance de la Diatomée Cyclotella ocellata semble régie par plusieurs facteurs dans la retenue El Kansera, en particulier la température, la disponibilité des nutriments (azotés), le broutage (zooplancton et 


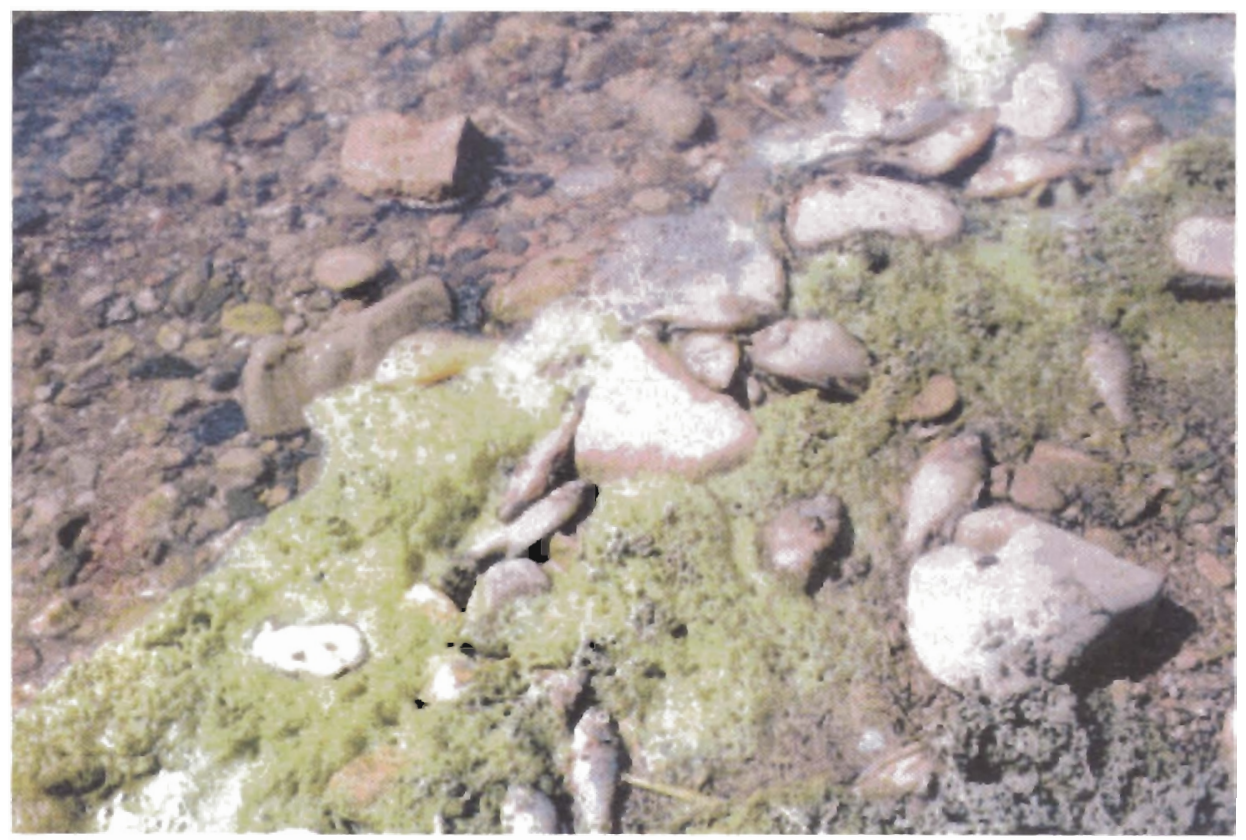

Photo 2. - Mortalité de Carpes de Chine sur les rives du lac-réservoir Photo 2. - Mortality of Chinese carp on the shores of the lake-reservoir

poissons planctonophages) et la compétition avec l'espèce Microcystis aeruginosa à caractère toxique reconnu (Thébault et al. 1995).

\section{CONCLUSION}

Le suivi du compartiment phytoplanctonique dans le lac-réservoir El Kansera met en évidence de profondes modifications d'une année à l'autre, dans la composition et la structure du peuplement:

- Durant l'année 1995 (année de sécheresse), l'azote est le facteur limitant; ce qui expliquerait la prédo- minance des Cyanobactéries en cette période, avec notamment $\mathrm{Mi}$ crocystis aeruginosa comme espèce dominante. Cette algue forme des blooms spectaculaires dans la retenue et semble être favorisée, en grande partie, par la grande stabilité du milieu (retenue à transit lent, Derraz 1995 et apports allochtones limités), ainsi que par les températures élevées et le fort ensoleillement de cette année sèche. De plus, la faible pressioil de broutage de cette algue par le zooplancton herbivore (effet de toxicité) stimulerait encore plus la formation de ces blooms. II est à noter, qu'au moment du bloom à Microcystis en juin 1995, une grande quantité de 
cadavres de poissons (Carpes argentées de Chine) a été observée sur les rives du lac-réservoir (voir photo 2), probablement en raison d'une intoxication par les microcystines (toxines) de cette algue. Plusieurs autres cas d'intoxication de poissons par l'espèce Microcystis aeruginosa ont été évoqués dans la littérature, notamment dans le lac Washington (EtatsUnis), dans le Neuse River en Caroline du Nord (Robert et al. 1986) etc...Cet effet de toxicité peut même représenter une "menace» pour l'être humain (Thébault et al. 1995), soit indirectement en consommant les poissons pêchés dans la retenue, soit directement en buvant l'eau du lac-réservoir (cas des villes de Tiflet et de Khemisset notamment qui se trouvent à quelques kilomètres de la retenue et qui sont alimentées en eau potable par ce barrage). Au centre d'hémodialyse du Brésil par exemple, des cas de décès ont été signalés en 1996 suite à une intoxication de l'eau par Microcystis aeruginosa.

L'année suivante, l'enrichissement du milieu en nitrates (pluies, lessivage du bassin versant et crues de l'oued Beht) est à l'origine d'une diversification spécifique algale et l'apparition de nouveaux groupes phytoplanctoniques, en particulier des Diatomées centriques (Cyclotella ocellata) et des Chlorococcales (Crucigenia apiculata, Scenedesmus ecornis...). Toutefois, en été, le relargage du phosphore par le sédiment associé aux températures estivales élevées, entraînent la réapparition de Microcystis aeruginosa dans le lac- réservoir. $\mathrm{Ce}$ qui représente un risque "potentiel » pour le consommateur.

Si l'on tient compte de ces résultats, l'état trophique du lac-réservoir El Kansera, déterminé à partir des associations phytoplanctoniques (Rosen 1981, Hutchinson 1967) et des paramètres de biomasses (Vollenweider 1971, OCDE 1982) évolue d'une année à l'autre; eutrophe à hypereutrophe durant l'année de sécheresse 1995, la retenue El Kansera devient l'année suivante oligo-mésotrophe, avec un retour à l'eutrophie en saison estivale. L'impact climatique semble ainsi jouer un rôle majeur dans l'évolution de la qualité des eaux de cette retenue.

\section{RÉFÉRENCES BIBLIOGRAPHIQUES}

Abdallaoui, A., Derraz, M., Benabdallah, M.Z., \& Lek, S. (1998). Contribution à l'étude de la relation entre les différentes formes du phosphore dans les sédiments d'une retenue de barrage eutrophe en climat méditerranéen (EI Kansera, Maroc). Rev. Sci. Eau, 1998 : 101-116.

Abouzaid, H. \& Foutlane, A. (1986). L'eutrophisation de quelques lacs-réservoirs au Maroc. Revue Marocaine du Génie Civil et des Travaux Publics., 9(83) : 35-39.

AFNOR (1994). In recueil des normes françaises, eaux, méthodes d'essais : $342 \mathrm{pp}$.

Capblancq, J. (1972). Phytoplancton et productivité primaire de quelques lacs d'altitude dans les Pyrénnées. Annal. Limnol., 8 : 231-321. 
Capblancq, J. (1974). Recherches sur la productivité primaire des lacs d'altitude. Doctorat es-sciences, Univ. Paul Sabatier, Toulouse.

Capblancq, J. (1982). Phytoplancton et production primaire. In "Ecologie du plancton des eaux continentales" Pourriot, R. Coll. Ecologie 16. Ed. Masson, Paris.

Cardinal, C. (1983). Composition et évolution saisonnière du phytoplancton du lac de Créteil (Val de Marne - France). Rev. Sc. Eau, $2: 153-172$.

Cherifi, O. (1992). Evolution et dynamique du phytoplancton en relation avec certains paramètres biotiques et abiotiques au niveau de la retenue Lalla Takerkoust (Maroc). DES du $3^{\text {ene }}$ cycle, Fac. Sc. Semlalia, Univ. Cadi Ayad, Marrakech, 155 pp.

Dauta A. (1982). Conditions de développement du phytoplancton. Etude comparative du comportement de huit espèces en culture. I- Détermination des paramètres de croissance en fonction de la lumière et de la température. Annls Limnol. 18(3) : 263-292.

Derraz, M. (1987). Influence des facteurs environnementaux sur l'évolution de la qualité des eaux de la retenue Oued El Makhazine (Maroc). Doctorat $3^{\text {eme }}$ cycle. Univ. Paul Sabatier de Toulouse : $95 \mathrm{pp}$.

Derraz, M. (1995). Etude de l'eutrophisation de la retenue de barrage El Kansera (Maroc). Caractéristiques physicochimiques, biodisponibilité du phosphate sédimentaire, écophysiologie de Microcystis et relation des blooms avec les paramètres environnementaux. Thèse d'Etat es-sciences à la Faculté de Meknès: $120 \mathrm{pp}$.

Devaux, J., Lair, N. \& Amblard, C. (1982). Un écosystème lacustre profond: le lac Pavin. In "Problèmes d'écologie: structure et fonctionnement des écosystemes limniques". LAMOTTE \& BOURLIERE, F. Ed. Masson Paris : 1 49.
Enrisken, H.P. \& Moestryp, O. (1997). Seasonal variation in microcystin contents of Danish Cyanobacteria. Nat. Toxins, 5(3) : 99-106.

Fqih Berrada, D., Berrada, R., Benzekri, M.A. \& Jabry, E. (1998). Dynamique des peuplements bactériens dans la retenue EI Kansera - relations trophiques avec le phytoplancton et le zooplancton - Acte au séminaire "Environnement, Etudes d'impact et développement humain durable", Settat (Maroc).

Fqih Berrada D., Berrada R., Benzekri A. \& Jabry E. (1999). Variations spatiotemporelles des peuplements de Cladocères, de Copépodes et de Rotifères dans la retenue El Kansera (Maroc). Acte au "Deuxième Colloque International sur l'Eau et l'Environnement ", Agadir (Maroc).

Fqih Berrada, D., Berrada, R., Benzekri, M.A. \& Fahde, A. (2000) Hétérogénéité horizontale des peuplements microphytoplanctoniques et zooplanctoniques dans la retenue El Kansera (Maroc) en relation avec les paramètres abiotiques. Revue des Sciences de l'Eau.13/3 : 213-236.

Gayral-Engerbaud, P. (1954). Recherches phytolimnologiques au Maroc. Thèse de Doctorat, Univ. Paris : 156 pp.

Hermann, H. (1980). Interesting planctonic forme littoral algae from waters in the district haule G.D.R. 3, Arch. Protistink, $123:$ 349-357.

Holmes, R. W. (1966). Light microscope observations on cytological manifestations of nitrate, phosphate and silicate deficiency in four marine centric diatoms. J. Phycol., $2: 136-140$.

Hutchinson, G. E. (dans Lair 1975) (1967). A treatise on limnology IIIntroduction to lake biology and the limnoplankton. John Wiley and Sons, inc, $115 \mathrm{pp}$.

Jrad, A. (1989). Phytoplancton et production primaire dans le lac de Pareloup 
(Aveyron). Thèse $3^{\text {ome }}$ cycle, Toulouse : $166 \mathrm{pp}$.

Köhler, J. (1992). Influence of turbulent mixing on growth and primary production of Microcystis aeruginosa in the hypertrophic bautzen reservoir. Arch. Hydrobiol. 123(4) : 413-429.

Konopka, A. E., Klemer, A. R., Walsby, A. E. \& Ibelings, B. W. (1993). Effects of macronutrients upon buoyancy regulation by metalimnetic Oscillatoria agardhii in Deming Lake, Minnesota. $J$. Plankton. Res., 15(9) : 1019-1034.

Le Cohu, R., Comoy, N., Guitard, J. \& Brabet, J. (1990). Périodicité saisonnière des diatomées planctoniques dans un grand réservoir: le lac Pareloup. Résumés des communications du $10^{\text {trmu}}$ colloque ADLAF, lle des Embiez.

Legendre, L. \& Watt, W.D. (1971). On a rapid technic for plankton enumeration. Ann. Inst. Oceanogr., Paris, 8 : 173-177.

Lewis, W.M. (1983). Interception of atmospheric fixed nitrogen as an adaptative advantage of scum formation in blue-green algae. J.phycol. $19: 534-$ 536.

Lohmann, H. (1908). Untersuchungen zur festellung des Vollstandigen gehaltes des Meeres an Plankton. Wiss Meeresunters, Abt. Kiet. N. L., $10: 132-170$.

Lorenzen, C.J. (1967). Determination of chlorophyll and pheopigments : spectrophotometric equations. Limnol. Oceanogr., 12 : 343-346.

Loudiki, M. (1990). Etude limnologique d'un hydrosysteme récemment aménagé dans la région de Marrakech (Maroc). Apports du bassin versant, hydrochimie, algues et macrophytes. Thèse d'Etat es-sciences, Faculté des Sciences et Techniques de Saint-Jérôme : $353 \mathrm{pp}$.

Loudiki, M., Oudra, B., Sbiyyaa, B. \& Mouhri, K. (1995). Inventaire et bioécologie des Cyanobactéries toxiques occasionnant des blooms dans divers plans d'eau douce du Maroc. Acte des Pre- mières journées Franco-Maghrébines sur les phycotoxines. Univ. Hassan II, Casablanca.

Moncef, M. (1993). Etude de la communauté zooplanctonique d'un écosystème lacustre peuplement des copépodes et des larves de Chaoboridés dans la retenue Al Massira (Maroc). Thèse Doct. D'Etat, Univ. Cadi Ayyad $180 \mathrm{pp}$.

O.C.D.E (1982). Eutrophisation des eaux: méthodes de surveillance, d'évaluation et de lutte. $O C D E$, Paris, $165 \mathrm{pp}$.

ONEP (1996). Rapport sur la lutte contre l'eutrophisation des lacs-réservoirs marocains, ONEP, Maroc, $16 \mathrm{pp}$.

Piersen, D.C. \& Weyhenmeyer, G.A. (1994). High resolution measurements of sediment resuspension above an accumulation bottom in a stratified lake. Hydrobiologia. 284 : 43-57.

Pont, D. (1983). Recherches qualitatives sur le peuplement de Copépodes, Cladocères et Ostracodes des rizières de Camargue. Thèse de Doctorat. Sc. Nat., Univ. De Provence, Marseille: $353 \mathrm{pp}$.

Porter, G.K. \& Orcutt, J.D.Jr. (1980). Nutritional adequacy, manageability and toxicity as factors that determinate the food quality of green and blue-green algae for Daphnia. Evolution and Ecology of zooplankton communities. Ed. W. Ch. Kerfoot.

Reynolds, C.S. (1984). Phytoplankton periodicity : the interactions of form, function and environmental variability. Freshw. Biol., 14(2) : 111-142.

Robert, R.C., Wade, L.B.Jr. \& Stanley, D.W. (1986). Toxic algal blooms in the Neuse River (North of Carolin). Report.10 pp.

Rosen, G.M. (1981). Phytoplankton indicator and their relations to certain chemical and physical factors. Limnol. (Berlin), 13(2) : 263-290. 
Sommer, U. (1989). Plankton ecology. Succession in plankton community. Springer-verlag, Berlin : $360 \mathrm{pp}$.

Sbiyyaa, B., Loudiki, M. \& Oudra, B. (1997). Capacité de stockage intracellulaire de l'azote et du phosphore chez Microcystis aeruginosa Kütz. et Synechocystis sp. Cyanobactéries toxiques occasionnant des blooms dans la région de Marrakech. Annls. Limnol., $34(3): 247-257$.

Testard, P. (1983). Le lac de Créteil : un lac de sablière peu profond. In "Problèmes d'écologie, Ecosystèmes limniques" Lamotte, M. \& Bourliere, F. (1983).

Thébault, L., Lesne, J. \& Boutin, J.P. (1995). Les cyanobactéries, leurs toxines et les risques pour la santé. Medecine trop. $55: 375-380$.

Varis, O. (1993). Cyanobacteria dynamics in a restored Finnish lake : a long term simulation study, Hydrobiologia, 268: 129-145.
Vollenweider, R.A. (1971). ( $1^{\text {tr }}$ Ed. 1968). Scientific fundamentals of the eutrophication of lakes and flowing waters, with particular references to phosphorus and nitrogen as factor in eutrophication. Tech.Report OECD, 192 p. $\left(2^{\circ} \mathrm{Ed}\right.$.).

Watanabe, M. F. \& Oish, S. (1983). A highly toxic strain of blue-green alga $\mathrm{Mi}$ crocystis aeruginosa isolated from lake Suwa. Bull. Jap. Soc. Fish/Nissuishi, 49(11) : 759-768.

Wetzel, R.G. (1983). Limnology. W. B. Saunders Campany Publishing, (Philadelphia), $767 \mathrm{pp}$.

Zhen-rong, H., Minjuan, Y. \& Jiawan, H. (1992). Toxins from Microcystis aeruginosa strain M. 8641. Biochem. J. 9(2) : 168-172.

Zohary, T. \& Robart, R.D. (1989). Diurnal mixed layers and long-term dominance of Microcystis aeruginosa. J. Plankton. Res., 11(1) : 25-48. 